\title{
Observación y experimentación en psicología: una revisión histórica
}

\section{Observation and experimentation in psychology: an historical revision}

\author{
Alma Gabriela Martínez \\ Antonio López-Espinoza* \\ Virginia Aguilera \\ Alma Galindo \\ Carolina De La Torre-Ibarra \\ Universidad de Guadalajara, México
}

Recibido: Mayo 30 de 2007

Revisado: J unio 14 de 2007

Aceptado: Julio 3 de 2007

\section{Resumen}

Se realiza una breve reseña histórica sobre las aportaciones teóricas y experimentales de diversos investigadores que contribuyeron al desarrollo de la psicología experimental. En la primera parte, se presenta una revisión de los inicios de la psicofísica y la psicología experimental. Posteriormente se caracterizan las aportaciones más importantes que involucraron los estudios en psicología animal, estructural y funcional, finalizando con la propuesta conductista de Watson y Skinner como método para estudiar los fenómenos psicológicos. Finalmente, se discute la utilidad de los estudios de laboratorio desde la perspectiva del análisis del comportamiento, enfatizando los alcances y desarrollo en Estados Unidos y diversos países de Latinoamérica.

Palabras clave: Psicología experimental, historia, desarrollo, estudio de la conducta.

\section{Abstract}

A brief history about theoretical and experimental contributions is described of researches to the development of the experimental psychology. In the

Esta investigación fue financiada por la Beca 180463 y el Proyecto 46083-H del CONACyT. Correspondencia: Dr. Antonio López-Espinoza. Centro de Estudios e Investigaciones en Comportamiento, Universidad de Guadalajara, Francisco de Quevedo \# 180, Col. Arcos Vallarta. C. P. 44130, Guadalajara, Jalisco, México. Correos electrónicos: Alma Gabriela Martínez e-mail: almagabrielamm@yahoo.com.mx, Antonio López-Espinoza e-mail: anton779@megared.net.mx. 
first part, a revision of the beginnings of the psychophysic and experimental psychology appears. Later, the most important contributions are characterized those studies that involved the studies in psychology animal, structural and functional, finalizing with the Watson and Skinner's behaviorist proposal as the method to study the psychological phenomena. Finally, we discussed the utility of the laboratory studies from the perspective of experimental analysis of behavior, emphasizing the reaches and development in the United States and diverse countries of Latin America

Key words: Experimental psychology, history, development, study of the conduct.

\section{Introducción}

Gracias a Galileo, los primeros métodos y resultados científicos aparecieron a partir del siglo XVII. Este científico añadió a los antiguos métodos de inducción y deducción la verificación sistemática a través de experimentos planificados. A finales del siglo XVII se amplió el uso de instrumentos de medición en la experimentación: el barómetro; la bomba de vacío, el péndulo, el microscopio, entre otros. Estas nuevas herramientas fueron útiles para el desarrollo y producción del conocimiento científico en diversas áreas científicas. Adicionalmente, la invención de la imprenta en 1440 hizo posible la producción masiva de libros, incrementando el efecto sobre el conocimiento mediante el lenguaje escrito como medio primordial para la comunicación.

La psicología, como cualquier otra área de conocimiento, empleaba técnicas especiales para cumplir con sus objetivos, sin embargo, éstas no consistían solamente en el uso de nuevos instrumentos de medición, sino en el desarrollo de la observación y experimentación. No obstante, el problema de la psicología radicaba en la definición conceptual de su objeto de estudio. Por esta razón, la investigación científica de los fenómenos psicológicos no podía restringirse al desarrollo de procedimientos para obtener "datos confiables", era necesario ir mucho más allá e instituir principios generales que contribuyeron a establecer una nueva ciencia.
Para que la psicología fuera reconocida como ciencia, fue necesario realizar una serie de trasformaciones a su marco teórico y experimental. El presente trabajo es un esfuerzo por hacer un breve recuento de las contribuciones de diversos investigadores que, implícita o explícitamente, colaboraron con el inicio y posterior desarrollo de la psicología como ciencia experimental. Se incluyeron pocos, pero fundamentales momentos históricos, que nos permiten caracterizar a la psicología como una disciplina científica.

\section{La psicofísica}

Diversos autores consideran que el comienzo formal de la psicología experimental se debe a Gustav Fechner. Este investigador fue el primero en realizar series experimentales bajo todo rigor científico, sus técnicas representaron la base de la metodología psicológica. Fechner estudió medicina, pero también tuvo la experiencia laboral necesaria para llamarse físico, filósofo, psicólogo y hasta poeta. Fechner estaba convencido que la filosofía debía estar basada en una sólida base científica (Benjamín, 1988; Boakes, 1984; Boring, 1990; Keller, 1990; Sahakian, 1987; Wozniak, 1999).

A partir de sus cátedras en física y matemáticas, Fechner se interesó por la relación entre estimulación física y sensación. Se hacía preguntas como: ¿qué tan brillante debe ser una estrella para que pueda ser observada?; 0, ¿qué tan fuerte 
debe ser un ruido para que se escuche? Diseñó diversas técnicas para encontrar respuestas precisas (Keller, 1990). Su principal obra: Elements of psychophysics, publicada en 1860, mostró claramente cómo podrían utilizarse procedimientos experimentales y matemáticos para estudiar la "mente humana" (Murchinson, 1930). Los métodos que utilizó fueron denominados psicofísicos y aunque pronto fueron reemplazados por nuevas técnicas más acordes a la psicología experimental, Fechner es considerado el precursor de la psicología experimental por tratar a los procesos psicológicos de la misma manera que las ciencias exactas y experimentales. Los estudios de Fechner fueron considerados experimentos, pues tomaba en cuenta los pasos básicos del método científico. Su propuesta le dio a la psicología la bienvenida al terreno científico.

\section{Los inicios de la psicología como ciencia experimental}

Definitivamente, fueron los fisiólogos como J ohannes Müller (con su teoría de la energía específica de los nervios), Hermann Von Helmholtz (el primero en medir la velocidad de los impulsos nerviosos) y Ernest Heinrich Weber (a quien Fechner le dio el crédito por la ley de Weber que sostiene que entre los estímulos y las intensidades de los estímulos hay una relación reglada) quienes iniciaron la investigación experimental en psicología. Sin embargo, fue Wilhelm Maximilian Wundt (alumno de Fechner por algún tiempo) el psicólogo que tiene el mérito de haber fundado el primer laboratorio para investigación psicológica. Su mayor logro fue introducir un estudio observacional activo y manipulativo para que la psicología convencional se convirtiera en una ciencia natural (Kantor, 1979).

Durante el periodo comprendido entre 1855 y 1919, Wundt dirigió 186 tesis que le otorgaron el título de "psicólogo decano de la historia de la psicología" en Leipzig. Wundt puso en funcionamiento su laboratorio psicológico en 1875, aunque la fecha de fundación exacta fue 1879. En 1881 publicó una revista que informaba resultados y métodos de in- vestigación en psicología. No obstante, es importante mencionar que antes de trabajar en Leipzig, Wundt realizó un amplio recorrido académico y científico por toda Alemania.

Una de las cualidades de Wundt para atraer estudiantes de diversas partes del mundo era la libertad para elegir sus temas de investigación. A los estudiantes no se les imponían temas y primero se publicaban sus investigaciones, para luego convertirse en tesis doctorales. El laboratorio de Wundt sirvió de modelo a gran cantidad de laboratorios de Europa y Estados Unidos. El trabaj o experimental que se llevaba a cabo en el laboratorio incluía: análisis de las sensaciones, procesos mentales, sentido del tiempo, atención, memoria y asociación de ideas. Para algunos historiadores Leipzig fue denominada como el laboratorio en psicología mejor equipado del mundo (Benjamín, 1988; Boring, 1990).

Wundt denominó a la psicología como la ciencia de la experiencia inmediata y sostenía que la fisiología era un instrumento para convertirla en ciencia (Wozniak, 1999). Utilizaba la introspección como método fundamental para obtener resultados. Para Wundt la introspección era "tener la experiencia" y "tener" era equivalente a "observar" en su vocabulario, como lo expresaba en algunas de sus publicaciones (Kantor, 1979).

Wundt mencionó que la observación era la única técnica adecuada para estudiar fenómenos psicológicos. Señalaba que para que una investigación pudiera ser exacta requería de control, de modo que era posible variar a voluntad numerosos factores que interactuaban con el objeto de estudio mediante técnicas experimentales (Keller, 1990).

Definitivamente, los esfuerzos de Wundt de enfatizar a la psicología fisiológica como ciencia trajeron como consecuencia la fundación de otros laboratorios para investigación experimental, la formación de nuevos científicos interesados en los fenómenos psicológicos y la clara afirmación que su método experimental "podía no ser tan adecuado para la observación de algunos fenómenos" (Titchener, 1869; 1921). 
Aunque Wundt tiene el mérito de iniciar con la investigación experimental, hubo otros importantes científicos que contribuyeron a establecer a la psicología como ciencia, tales como Hermann Ebbinghaus (convencido de que el experimentalismo era necesario para la psicología), Oswald Kûlpe (afirmó que los fenómenos no observables no existían para la ciencia); y por supuesto, el inglés Edward Bradford Titchener (Boring, 1990).

\section{La psicología estructural}

Con la publicación de su libro Experimental Psychology en 1901, Titchener buscaba que la psicología fuera considerada una ciencia respetable. Su trabaj o como científico lo desarrolló en Cornell, lugar donde propuso la psicología estructural (Boakes, 1984; Boring, 1990; Keller, 1990). Definió a la psicología como la ciencia de la mente, que a su vez fue definida como la suma total de la experiencia humana. Titchener (1921) intentó fortalecer el significado del término introspección, pues estaba convencido de que los términos científicos deberían ser utilizados con sumo cuidado e inequívocamente. Es probable que fuera de los primeros científicos en advertir la peligrosidad de utilizar significados de "sentido común" a los términos científicos.

Titchener creía que los datos primarios de la psicología debían obtenerse por medio de la introspección y bajo estrictas condiciones experimentales. Actualmente, es probable que estos datos fueran denominados como «subjetivos», pero sin duda, Titchener los señalaba como puramente objetivos (Boring, 1953).

Murchinson (1930) mencionó que entre los estudiantes más destacados de Titchener se encontraba Margaret Floy Washburn, la primera mujer en obtener un doctorado en psicología en Estados Unidos. Washburn publicó el libro The animal mind, convencida de que la investigación psicológica con animales era importante para el desarrollo de la psicología como ciencia. Además estableció su propio laboratorio para estudios psicológicos en 1903, en los que después de algunos años de trabaj o tra- tó de conciliar los postulados del conductismo con el método introspectivo.

\section{La psicología animal}

El lugar que tiene Ivan Petrovich Pavlov en la psicología es fundamental. Su interés profesional por los fenómenos psicológicos inició cuando tenía 50 años (Boakes, 1984). Su mayor aportación a la psicología animal fue el concepto de reflejo condicionado, lo que lo convierte indiscutiblemente en una las figuras más importantes del análisis de la conducta. Su calidad como científico es difícil de repetir: manejaba con cautela y diplomacia las diferentes teorías y tenía plena confianza en el trabajo de laboratorio. El supuesto general de Pavlov era que las actividades del sistema digestivo eran producto de arcos reflejos. En 1924 impartió varias conferencias que conformaron posteriormente su libro Conditioned reflexes. Después de varias investigaciones determinó que había relaciones espacio-temporales entre estímulos. Señaló que la conducta no es más que una cadena de reflejos, algunos innatos y la mayor parte aprendidos, adquiridos o condicionados por el simple hecho de haber sido asociadas (en algún momento de la existencia del organismo) con ciertas condiciones ambientales. Sus investigaciones Ilegaron a influir decisivamente en la fundación y desarrollo del conductismo (Boakes, 1984; Keller, 1990; Wozniak, 1999).

Por otro lado, la teoría de la evolución permitió la consolidación de la psicología animal. Con la publicación del libro Expression of the emotions in man and animals en 1872, Charles Darwin describió numerosas observaciones y afirmó que el comportamiento del hombre dependía de la herencia de ciertos comportamientos que fueron útiles en su vida animal anterior. En 1790 Erasmo Darwin, su abuelo, publicó su Teoría de la metamorfosis de las partes, en la que aseveraba que nuevas formaciones en las plantas pueden surgir a partir de simples modificaciones de sus antecesoras (Boring, 1990).

Siguiendo su tradición familiar, Charles Darwin se entrenó como un gran observador de animales y 
publicó su obra más importante, The origin of species, en 1859. Su teoría tuvo una gran influencia para la psicología de su época, pues señalaba que los animales tenían emociones que eran descritas como actitudes conductuales cualitativamente semej antes a las del hombre. Además utilizó una nueva terminología para referirse a los eventos conductuales que observaba una y otra vez. Los psicólogos contemporáneos consideraron válida la extrapolación de resultados obtenidos en investigaciones con animales para ser trasladados a la psicología humana. Uno de estos psicólogos fue George J ohn Romanes, considerado el fundador de la psicología comparativa (Benjamín, 1988).

Finalmente, la psicología evolutiva de Darwin proporcionó los fundamentos para la psicología estadística de Francis Galton (considerado el fundador de la Psicometría), Karl Pearson (que presentó a la comunidad científica el coeficiente de correlación) y Charles Spearman (fundador del análisis factorial); y, a la psicología de las diferencias individuales de J ames McKeen Catell (quien introdujo la estadística a la psicología norteamericana), que a su vez, marcaría el inicio de la psicología funcional en América.

\section{La psicología funcional}

Keller (1990) mencionó que la primera generación de psicólogos americanos que trataron de establecer a la psicología como ciencia se conformaba de cuatro grupos: 1) la psicología funcional de Catell; 2) la psicología evolutiva de Hall y Baldwin; 3) la psicología comparativa de Thorndike; y, 4) el conductismo de Watson.

La escuela funcionalista inició en la Universidad de Chicago en manos de John Dewey, James Rowland Angell y Harvey Carr. Los funcionalistas se oponían al estructuralismo de Titchener argumentando que el interés de la psicología no estaba en determinar qué son los procesos mentales, sino para qué sirven. No utilizaban la terminología científica y tenían "más libertades" en cuanto al minucioso y sistemático trabajo de laboratorio. Los funcionalistas iniciaron sus propuestas a partir de los trabaj os de William James, quien en su libro Principles of psychology, publicado en 1890, se oponía radicalmente a la psicología de Wundt. El objeto de estudio era la actividad mental y los métodos para estudiarla se conformaban por la observación subjetiva (introspección) y la observación objetiva (Dewey, 1884).

En la Universidad de Columbia surgieron otras versiones de la psicología funcional. Catell, alumno de Titchener por algún tiempo, se opuso al uso de la introspección para medir ciertos eventos psicológicos. Argumentaba que la investigación en animales debería centrarse a los aciertos y desaciertos que comete un sujeto de estudio en determinada tarea (Catell, 1928).

Columbia también fue el lugar de trabajo de Edward Lee Thorndike (su director de tesis doctoral fue Catell). Publicó su tesis Animal intelligence: an experimental study of the associative processes in animals en 1898. El objetivo general de sus estudios era evaluar el aprendizaje de perros y gatos. Fue el creador de la caja-problema en la que estudiaba el proceso de aprendizaj e por ensayo y error. Su procedimiento (poner al animal dentro de la caja y medir la latencia de la respuesta de salir) permitió obtener una situación experimental medible que posibilitaba comparaciones entre las respuestas de perros y gatos. A partir de sus estudios estableció la famosa Ley del efecto y se convirtió en la excepción de los psicólogos americanos que estudiaban la conducta animal, pues la mayoría se dedicaba al estudio de insectos (Thorndike, 1910).

Finalmente, Stanley Hall y J ames Mark Baldwin fueron dos psicólogos influenciados por el efecto de la teoría de Darwin. A partir de algunos preceptos de la teoría darwinista, establecieron laboratorios de psicología en Universidades como Clark, J ohn Hopkins y Princeton. Defendieron la postura de que las nuevas teorías psicológicas debían abarcar la explicación de un mayor número de fenómenos psicológicos. Adicionalmente, Baldwin fundó el Psychological Review en 1894, y diez años después, el Psychological Bulletin junto a Catell (Boring, 1990; Wozniak, 1999). 


\section{El conductismo}

J ohn Broadus Watson realizó su doctorado en la Universidad de Chicago y en 1903 obtuvo su grado. Su tesis doctoral fue dirigida por Angell y se tituló Animal education: the psychical development of the white rat. Boakes (1984) describió a Watson en su época de estudiante como un joven que disfrutaba manipular a las ratas de laboratorio, que tenía habilidades manuales para el manejo de instrumental y que iba trabajar todos los días de la semana sin descanso. Agregó que utilizaba ratas privadas de sus sentidos y las exponía a laberintos con diferentes niveles de complejidad y que Watson siempre enfatizaba en sus resultados la importancia del control experimental. Aunque algunos historiadores de la psicología afirmaron que con el trabajo de Watson el funcionalismo adoptó la forma del conductismo, la investigación psicológica de Watson dio como resultado un nuevo enfoque para la psicología como ciencia (Boring, 1990; Keller, 1990).

En 1913 Watson fundó el conductismo con su artículo Psychology as the behaviorist views it publicado en el Psychological Review. Y desde entonces se conoció a la ciencia de Watson como la psicología del estímulo-respuesta. Su primer libro publicado en 1914: Behavior, an introduction to comparative psychology, contenía numerosos párrafos dedicados a la importancia del trabajo en laboratorio: "La psicología como la ve el conductista es una rama experimental y puramente objetiva de las ciencias naturales". La psicología va a estudiar la conducta como su tema fundamental; su método va a ser enteramente objetivo y su problema central va a ser la predicción y el control (Watson, 1914). En otra de sus publicaciones: Psychology from the standpoint of behaviorist, declaró que no existía distinción alguna entre la psicología animal y la humana, sustentando esta afirmación en la teoría de la evolución de Darwin (Watson, 1919). En 1924 publicó su famoso libro Behaviorism, en el que explícitamente describía cómo diversos procesos psicológicos podían ser estudiados desde la perspectiva conductista. Para esa época Watson ya estaba más ocupado en el trabajo sistemático con humanos que con la experimentación en animales.

El objetivo general del movimiento conductista de Watson era definitivamente subrayar que la psicología era una ciencia si se especificaba claramente su objeto de estudio y se empleaban correctamente métodos adecuados (Harzem, 1993). Señaló que en las otras ciencias, los hechos de la observación son objetivos, verificables y pueden ser reproducidos por todos los observadores entrenados. Si la psicología era considerada como una ciencia de la "conciencia" no tendría tal cantidad de datos, pues no puede compartirlos, ni las otras ciencias pueden emplearlos. Determinó que aquellos eventos que no son obj etivamente verificables no pueden ser estudiados por una ciencia que presume de serlo (Watson, 1924).

Watson (1914) definió la psicología como "esa división de las ciencias naturales que toma como tema central a la conducta humana". Para Watson, la conducta consistía en respuestas, reacciones 0 aj ustes de un organismo a ciertos sucesos antecedentes (estímulos o situaciones de estímulo). Watson (1924) describió en qué consistía la experimentación para un conductista. Señaló que un experimento debía iniciar con la identificación precisa de los estímulos participantes y de la posible medición de respuestas específicas que emitirían los organismos. Indicó que sólo cuando aislamos, repetimos y variamos las condiciones de una observación sistemáticamente (cuando realizamos un experimento dentro de un laboratorio) podemos llegar a alguna expresión definida y cuantitativa del evento conductual (Watson, 1914).

\section{La psicología es una ciencia}

Reynolds (1973) señaló que la investigación experimental implica un redescubrimiento y una confirmación de hechos y principios anteriormente establecidos, pero también, una búsqueda de hechos y principios mal entendidos. Para que la psicología se convirtiera en una ciencia tuvo que redescubrir hechos, métodos y principios. Definitivamente, las aportaciones de Watson tuvieron con- 
secuencias positivas para la psicología y aunque fue blanco de numerosas críticas, convenció a la mayoría de los beneficios que obtendría la psicología al adoptar la perspectiva conductual.

El objetivo de la nueva ciencia era complejo pues debía interpretar un mundo no observable directamente, elegir los eventos sujetos a observación y analizar la interacción entre una conducta determinada y el ambiente en el que ocurre. Después de Watson, los principales conductistas formularon nuevas técnicas y procedimientos para analizar los fenómenos psicológicos más variados: el lenguaje, la percepción, el aprendizaje, el pensamiento, las emociones, etcétera, siempre desde una visión objetiva, y por lo tanto, científica. Keller (1990) señaló que después de 1920 los psicólogos comenzaron a debatir sobre la ciencia del laboratorio. Entre los investigadores más conocidos dentro de este periodo está E. R. Guthrie, que con su teoría de la contigüidad realizó un gran número de investigaciones experimentales en las que afirmaba que un estímulo que acompañó a un movimiento tenderá a repetirse si es seguido por ese movimiento (Guthrie, 1946).

Una investigación de laboratorio más elaborada fue la de Edward Tolman. Realizó una gran cantidad de experimentos con ratas de laboratorio en laberintos. Tolman (1922) trató de construir su sistema de aprendizaje sobre datos de experimentación. El aprendizaj e para Tolman consistía en cambios en el "conocimiento" del medio, de qué conduce a qué, de qué será recompensado o castigado bajo determinadas circunstancias. Argumentó que en el laberinto, la rata desarrollaba un "mapa cognoscitivo", más que una serie de movimientos específicos. La ejecución del animal representaba su aprendizaje con el objetivo de alcanzar una meta (Catania, 1975). Aunque fueron varios los que criticaron los términos de Tolman, su trabajo experimental sirvió de base para otros estudios (Boakes, 1984).

A partir de los trabajos de estos investigadores, la psicología norteamericana creció rápidamente. Los psicólogos-investigadores eran altamente reconocidos ocupando importantes puestos en universi- dades. El estudio de la psicología animal tuvo al principio una posición menor, pero posteriormente, la mayoría de los laboratorios contaba con ratas blancas para experimentación. En definitiva, la psicología progresó rápidamente y la psicología experimental alcanzó el apogeo gracias al conductismo, lo que dio origen a los trabajos de nuevos investigadores que iniciaron nuevas áreas de investigación, como Curt Richter, Clark Leonard Hull y Robert Mearns Yerkes. Adicionalmente, el conductismo permitió la aparición de nuevos marcos teóricos y conceptuales que dieron origen a las ciencias del comportamiento, como el análisis experimental de la conducta.

Richter se graduó en Harvard y se desempeñó como asistente en el departamento de Filosofía y Psicología. Posteriormente conoció al profesor Robert Yerkes con quien trabajó afanosamente sobre la conducta de insectos en la Universidad J ohn Hopkins. En repetidas ocasiones, Yerkes le dio a leer el manifiesto conductual y otros trabajos de Watson sobre el estudio de la conducta de los animales. Sin embargo, Richter no se mostró muy interesado en las ideas de Watson sobre el conductismo, su interés radicó, más bien, en la importancia que Watson le daba a la conducta como campo de investigación científica. Curiosamente, cuando Richter llegó a la Universidad J ohn Hopkins, no conincidió con Watson, a pesar de que llegó a ocupar su cargo en el laboratorio.

La fama de Richter se debe en gran medida, a su habilidad para el desarrollo de métodos para cuantificar diversas conductas. Estos métodos se caracterizaban por su simplicidad y facilidad para ponerlos en práctica. Adicionalmente, diseñó numerosos instrumentos y equipos con los que trabajó por casi 60 años.

No obstante, sus estudios sobre la función autorreguladora, en los que señalaba que "la conducta del organismo contribuye definitivamente en el mantenimiento de estados estables internos" (Richter, 1922, 1943), propiciaron al inicio y desarrollo de importantes líneas de investigación relacionadas con apetitos específicos por: a) sabores (Capaldi, 1996); b) diversas concentraciones de 
sodio (Contreras y Frank, 1979); c) venenos (Garcia, Hankins y Rusiniak, 1976); y, d) todo lo relacionado con la conducta de selección de nutrientes y dietas (Sclafani, 1990).

Por su parte, el trabajo de Hull es completamente distinto a cualquier otro teórico conductual. Bower y Hilgard (1989) califican la obra de Hull como "el mejor ejemplo de la teorización sistemática en psicología durante la primera mitad del siglo XX". Orgulloso de su formación conductista, Clark Hull (graduado por la Universidad de Michigan y enfermo de poliomielitis desde los 24 años) nunca utilizaba el término de conciencia en sus artículos y disertaciones. Era un gran admirador del trabajo de Pavlov y sus reflejos condicionados y de Thorndike y su ley del efecto.

Sus principales construcciones teóricas fueron la fuerza del hábito, el nivel del impulso y sus postulados sobre la inhibición. Hull (1934a, 1934b) designó como fuerza de hábito a la fuerza de la asociación entre un estímulo y una respuesta. Asimismo, dio una gran importancia al término de impulso en la mayoría de sus publicaciones, pues manifestaba que sin impulso no puede haber reforzamiento, respuesta o regulación de hábitos. Señaló que el estado de necesidad o de impulso especifica el objetivo que va a reducir o a terminar con la necesidad, por lo que el impulso determina la fuerza de la respuesta, la acción del reforzador y la asociación entre los estímulos y la respuesta. Adicionalmente trabajó con la inhibición condicionada de Pavlov, sin embargo, sus estudios al respecto fueron duramente atacados. Hull (1953) mencionó en uno de sus últimos escritos que su mayor contribución a la Psicología fue la de "intentar dar una explicación cuantitativa y sistemática de algunas de las más importantes formas de conducta social no humana".

Para otros, el mayor mérito de Hull fue darle al aprendizaje una nueva visión conductista y mecaniscista, lo que propició nuevos preceptos sobre el tema y numerosas confrontaciones con personajes de alta envergadura que no concordaban con sus postulados, como fue el caso de Tolman (Benjamín, 1988; Hearst, 1979). Las confrontacio- nes entre estas grandes figuras encontraron su punto más álgido en 1934, durante un debate sobre el aprendizaje organizado en la reunión anual de la American Psychological Association (APA) en la Universidad de Columbia. Tolman criticó duramente la teoría del reflejo condicionado, por lo que Hull organizó una mesa redonda para atacar la falta de lógica de la teoría de Tolman, plagada de términos cognoscitivos. Al día siguiente, Tolman retomó la pelea y junto a Neil Miller presentó dos experimentos en defensa de su posición sobre la adquisición del aprendizaje.

Posteriormente, Hull (1953) continuó defendiendo su teoría sobre la fuerza del hábito. En esta ocasión, su mayor mérito fue el apego a una estricta perspectiva conductista, pues intentó especificar detalladamente la interacción entre las variables experimentales que participan en un experimento. En definitiva, Hull fue un ej emplo a seguir respecto a la sistematización del esquema conductual para obtener resultados experimentales.

Finalmente, es importante hacer un breve recuento del trabajo de Yerkes respecto a la psicología comparada. Boring (1990) señaló que este autor puede ser considerado como el líder del movimiento norteamericano de la psicología comparada, debido a su persistencia y forma en la que extendió su influencia en la organización y sistematización científica. Realizó experimentos con gran cantidad de especies, empezando sus estudios con insectos, seguido del estudio de palomas, cerdos, monos, ratas, entre otros, para terminar con estudios respecto a la conducta del hombre. Entre sus mayores contribuciones destaca la fundación del primer laboratorio especializado en la investigación de primates en Estados Unidos, denominado "Yerkes Nacional Primate Research Center".

\section{Skinner y la modificación de la conducta}

Uno de los conductistas que hoy en día sigue conservando un gran número de seguidores es Burrhus Frederik Skinner. "Fred" (como le gustaba que le Ilamaran), o profesor Skinner (como le llamaba la 
mayoría) ingresó a la Universidad Hamilton College, se licenció en literatura inglesa y estudió un diplomado en lenguas románicas. Estudió y obtuvo su doctorado en Psicología en la Universidad de Harvard, en donde trabajó hasta 1936. Posteriormente dirigió el departamento de Psicología en la Universidad de Indiana, pero regresó a Harvard en 1948 para quedarse ahí el resto de su vida haciendo investigación.

Siendo estudiante de la Universidad de Harvard inventó la caja que lleva su nombre para facilitar el estudio de la alimentación de las ratas. Sus estudios dieron como resultado la división del proceso de aprendizaje en respuestas operantes y estímulos reforzantes. Prácticamente basó sus teorías en el análisis de las conductas observables. Su teoría condujo al desarrollo de técnicas de modificación de conducta aplicadas a programas de enseñanza-aprendizaje en el aula.

Ribes (1991) mencionó que para Skinner, la conducta era un fenómeno ordenado. Ése fue su punto de partida para diseñar grandes avances tecnológicos en materia experimental que contribuyeron enormemente a la organización y rigor científico en la psicología posterior. El registro acumulado de frecuencias, la técnica de la operante libre y los diseños de replicación intrasujetos representan posiblemente sus grandes aportaciones al campo experimental de la psicología.

\section{El debate del laboratorio: Conclusiones}

Actualmente, y desde hace algunos años, el debate científico sobre la utilidad del laboratorio se ha retomado con más fuerza. Diversos autores han discutido la confiabilidad de los estudios en laboratorio, señalando que la naturaleza en sí misma es caprichosa y que la forma en que los organismos se conducen no tiene por qué ser ordenada, lo que dificultaría cualquier enfoque científico de la conducta, excluyendo la posibilidad de análisis de la naturaleza animal y humana (Woods y Seeley, 2002). Si bien es cierto que pueden Ilevarse a cabo investigaciones serias fuera del laboratorio, como la mayoría de los estudios sobre aprendizaj e en ni- ños, no hay que olvidar que la investigación más sencilla es aquella en la que se controla el mayor número de variables posibles. Como ciencia del comportamiento, el análisis experimental de la conducta se propone demostrar la validez de su tratamiento controlando un evento específico y se aparta de aquellos eventos que se efectúan en situaciones no controladas. El suj eto de estudio dispone de un espacio experimental específico, reducido a un número de estímulos y respuestas observables y manipulables (Catania, 1975; Ferster, 1953). Con base en esta postura, Perone (1991) y Premack (1959) afirmaron que la variedad y complejidad de la conducta puede estudiarse en condiciones reducidas y se hace posible observar un número limitado de fenómenos. Sidman (1960) señaló que un experimento científico debe obtener sus resultados a partir de la manipulación de variables del propio experimentador, subrayó que la característica más importante de un experimento es el control.

Harzem (1996) mencionó que la conducta puede ser controlada accidental o deliberadamente por la naturaleza, por el hombre, por su cultura, o sus propias instituciones sociales. Es decir, por una variedad de factores que interactúan constantemente. Mencionó que desde este enfoque, un experimento consiste en hacer una pregunta específica y situarla en la naturaleza. Adicionalmente, el diseño experimental en conducta operante debe guiarse conforme a reglas que la propia práctica científica proporciona (Ferster, 1953). El trabajo de laboratorio es y sigue siendo fundamental. La discusión, definitivamente, no puede concentrarse en definir si la naturaleza puede reducirse en un laboratorio, sino en la especificación de las variables experimentales a estudiar. El hecho de reconocer la falta de orden en la emisión de conductas no es afirmación suficiente para evadir la responsabilidad de examinar un evento conductual de interés científico.

No hay forma de definir el objeto de estudio de la psicología de una manera que satisfaga a todos a los psicólogos, pues cualquier definición puede resultar demasiado exclusiva. Además, la falta de distinción entre lo científico y lo técnico se ha pro- 
nunciado en los últimos años, lo que ha traído consecuencias importantes. No todas son negativas, pues ha permitido tener más control de las variables psicológicas que son objeto de estudio y una mejor cuantificación de lo que medimos. No obstante, la complejidad de los fenómenos, en algunos casos, se aborda sin la rigurosidad de la sistematización científica (Marx y Hillix, 1973).

Los experimentos que se llevan a cabo desde la perspectiva de la ciencia del comportamiento, 0 más específicamente, desde el análisis experimental de la conducta, se caracterizan por la clara identificación de la relación entre la conducta y su medio que se pretende estudiar y por la finalidad de encontrar cambios reales, exactos y específicos de la conducta de los organismos.

Para el psicólogo conductual, dos de los acontecimientos conductuales fundamentales, estímulo y respuesta, pueden combinarse de innumerables maneras. Su estudio debe caracterizarse por la elección de una sola combinación en un determinado momento, pues el control experimental es una de las características más importantes en un experimento. Gran parte de la investigación científica actual se elabora dentro de un laboratorio, pues ahí es donde puede controlarse el mayor número de variables mediante aplicaciones tecnológicas. Es posible que fuera del laboratorio puedan observarse conductas "más fieles" a la naturaleza animal y humana, pero no hay que olvidar que la conducta presenta un alto grado de complejidad que hace imposible hacer una descripción simple en escenarios naturales. A medida que ha aumentado la capacidad de análisis se han estudiado conductas cada vez más complejas, bajo contingencias que se aproximan a la sutilidad y complej idad de las contingencias que hallamos en el ambiente cotidiano.

Los psicólogos experimentales trabaj an actualmente en una gran variedad de tópicos, se han preocupado por la creación de centros de investigación que respetan rigurosamente el método científico. Adicionalmente, la tecnología utilizada ha progresado en gran medida: los métodos de registro y estimulación han llegado a un alto nivel de exactitud y los laboratorios contienen instrumentos electrónicos que presumen del mayor refinamiento tecnológico. Todo ello gracias a la intervención de la electrónica e ingeniería, pero siempre favorecidas por las inquietudes de un psicólogo dispuesto a evaluar una nueva variable.

Los resultados de esta combinación de intereses conductuales, tecnología y disposición ha provocado la creación de diversas asociaciones profesionales de Psicología. Muchas de ellas cuentan con divisiones especializadas en el estudio de la Psicología Experimental. Quizá la más importante es la American Psychological Association (APA por sus siglas en inglés) que tiene la división de: 1) Psicología Experimental; 2) Psicología Fisiológica; 3) Psicología Comparada; y, 4) Análisis Experimental del Comportamiento.

De igual forma, se lleva a cabo la publicación de resultados en diversas revistas. Entre las publicaciones de mayor alcance se encuentran: 1) J ournal of Experimental Psychology: Animal Behavior Processes; 2) Quarterly Journal of Experimental Psychology; 3) Journal of the Experimental Analysis of Behavior; 4) Animal Behavior; 5) Behavioral and Brain Sciences; 6) British Journal of Psychology; 7) Learning and Motivation; 8) Physiology and Behavior; 9) Journal of Behavior Analysis and Therapy; 10) Psychological Bulletin; $y, 11)$ Psychological Review.

En México también se cuenta con revistas especializadas en el tema del estudio de la conducta como la Revista Mexicana de Análisis de Ia Conducta, fundada en 1975. Adicionalmente, se lleva a cabo el Congreso Mexicano de Análisis de la Conducta desde 1974, organizado por la Sociedad Mexicana de Análisis de la Conducta (SMAC). Dicho evento fue auspiciado en la década de los setentas por la Facultad de Psicología de la Universidad Veracruzana y por la Facultad de Psicología de la Universidad Nacional Autónoma de México (UNAM) (Ribes, 2006). Actualmente diversas universidades públicas y privadas del país incluyen dentro de sus programas la temática básica de la psicología como ciencia del laboratorio. De igual forma se ofrece un posgrado con clara orientación conductual: la 
Maestría y Doctorado en Ciencias del Comportamiento, opción Análisis de la Conducta de la Universidad de Guadalajara, México.

Por su parte, en Brasil existen varias asociaciones y se hacen numerosas publicaciones, entre ellas la revista Modificação do Comportamento. En el Perú hay varios grupos trabajando activamente y se publica la Revista Peruana de Análisis de la Conducta. Por su parte en Colombia, la Pontificia Universidad J averiana de Bogotá publica Universitas Psicológica y la Universidad Santo Tomás, la Revista Diversitas Perspectivas en Psicología en las que se incluyen estudios sobre la modificación de conducta. Incluso en Argentina y Uruguay también se trabaja activamente en procedimientos de modificación del comportamiento y en investigación básica.

Definitivamente, el desarrollo y alcance de la psicología científica no sólo descansa en los avances tecnológicos en el área o en la creación de centros especializados para el estudio de la conducta, se dirige a la relevancia social y aplicaciones prácticas de los hallazgos en el laboratorio. Todavía queda mucho por hacer, sobre todo en la cuestión teórica y en el desarrollo de diseños novedosos y confiables para la búsqueda de nuevas interrogantes.

No olvidemos que para describir la conducta se involucran diversos esquemas conceptuales que en numerosas ocasiones no son los más adecuados. Sin embargo, aún no es posible emplear una terminología estrictamente científica, una correcta descripción de una conducta puede efectuarse si logramos identificar qué observamos, qué medimos y qué obtenemos en un espacio temporal determinado.

\section{Referencias}

Benjamín, L. T. (1988). A history of psychology. Nueva York: McGraw Hill Inc.
Boakes, R. A. (1984). Historia de la psicología animal: de Darwin al conductismo. Madrid: Alianza Editorial.

Bower, G.H. y Hilgard, E.R. (1989). Teorías del aprendizaje. México: Trillas.

Boring, E. G. (1953). A history of introspection. Psychological Bulletin, 50, 169-189.

Boring, E. G. (1990). Historia de la psicología experimental. México: Trillas.

Capaldi, E. D. (1996). Conditioned food preferences. En E. D Capaldi (ed). Why we eat what we eat. USA: American Psychological Association, 53-80.

Catania, A. C. (1975). Investigación contemporánea en conducta operante. México: Trillas.

Cattell, J. M. (1928). Early psychological laboratories. Science, 67, 543- 548.

Contreras, R.J. y Frank, M. (1979). Sodium deprivation alters neural responses to gustatory stimuli. Journal of General Physiolog, 73, 569-594.

Dewey, J. (1884). The new psychology. Andover Review, 2, 278-289.

Ferster, C. B. (1953). The use of the free operant in the analysis of behavior. Psychological Bulletin, 50, 263-274.

García, J., Hankins, W. G. y Rusiniak, W. (1976). Behavioral regulation of the milieu interne in man and rat. Science, 185, 824-831.

Guthrie, E. R. (1946). Psychological facts and psychological theory. Psychological Bulletin, 43, 1-20.

Harzem, P. (1996). The craft of understanding the mind: Why can not be a science. Revista Mexicana de Análisis de la Conducta, 2, 5-26. 
Hearst, E. (1979). The first century of experimental psychology. Nueva Jersey: Lawrence Erlbaum association publishers.

Hull, C. L. (1934a). The concept of the habit-family hierarchy and maze learning: Part I. Psychological Review, 41, 33-54.

Hull, C. L. (1934b). The concept of the habit-family hierarchy and maze learning: Part II. Psychological Review, 41, 134-152.

Hull, C. L. (1953). A behavior system: an introduction to behavior theory concerning the individual organism. New Haven, CT: Yale University Press.

Kantor, J. R. (1979). Wundt, experimental psychology and natural science. Revista Mexicana de Análisis de la Conducta, 5, 117-129.

Keller, F. S. (1990). La definición de psicología. México: Trillas.

Marx, M. H. y Hillix, W. A. (1973). Systems and theories in psychology. The American J ournal of Psychology, 86, 886-887.

Murchinson, C. (1930). History of psychology autobiographic. Worcester: Clark University Press.

Perone, M. (1991). Experimental design in the analysis of free-operant behavior. En I. $\mathrm{H}$ Iversen y K. A Lattal (eds.) Experimental analysis of behavior. Part 1. Elsevier: Nueva York: 135- 171.

Premack, D. (1959). Toward empirical behavior Iaws: I. Positive reinforcement. Psychological Review, 66, 219-233.

Reynolds, G. S. (1973). Compendio de conducta operante. México: Editorial Ciencia de la Conducta S.A.

Ribes, E. (1991). Psicología general. México: Trillas.

Ribes, E. (2006). Antecedentes y desarrollo histórico. En CEIC XV aniversario Memorias 1991-
2006. Universidad de Guadalajara, Centro Universitario de Ciencias Biológicas y Agropecuarias, 15-40.

Richter, C. P. (1922). A behavioristic study of the activity of the rat. Comparative Psychology. Monographies, 1, 1-55.

Richter, C. P. (1943). Total self regulatory functions in animals and human beings. Harvey Lecture Series, 38, 63-103.

Sahakian, W. S. (1987). Historia y sistemas de la psicología. Madrid: Tecnos, S. A.

Sclafani, A. (1990). Nutritionally based learned flavor preferences. En E. D. Capaldi, y T. L. Powley (eds.). Taste, experience $\&$ feeding: development and learning. USA: American Psychological Association.

Sidman, M. (1960). Tactics of scientific research: Evaluating experimental data in psychology. Nueva York: Basics Books.

Thorndike, E. L. (1910). The contribution of psychology to education. Journal of Educational Psychology, 1, 5-12.

Titchener, E. B. (1869). A psychological laboratory, Mind, 7, 311-331.

Titchener, E. B. (1921) Brentano and Wundt: Empirical and experimental psychology. American J ournal of Psychology, 32, 108-120.

Tolman, E. C. (1922). A new formula for a behaviorism. Psychological Review, 29, 44-53.

Watson, J. B. (1914). Psychology as the behaviorist views it. Psychological Review, 20, 58-177.

Watson, J. B. (1919). Psychology from the standpoint of a behaviorist. Philadelphia: J. B. Lippinicot Co.

Watson, J. B. (1924). Behaviorism. New York: Norton. 
Woods, S. C. y Seeley, R. J. (2002). Hunger and energy homeostasis. En R. Gallistel y H. Pashler (eds). Steven's handbook of experimental psychology. Nueva York: John Wiley $\&$ son's, 633-668.
Wozniak, R. H. (1999). Classics in psychology, 18551914: Historical essays. Bristol: Thoemmes Press. 